\section{PTH-066 CHOLANGIOSCOPY-ASSISTED ELECTROHYDRAULIC LITHOTRIPSY IS HIGHLY EFFECTIVE IN THE MANAGEMENT OF DIFFICULT BILE DUCT STONES}

doi:10.1136/gutjnl-2013-304907.553

1." V P K Lekharaju, ${ }^{1} \mathrm{O}$ Noorullah, ${ }^{2} \mathrm{M}$ Kumar, ${ }^{1} \mathrm{C}$ A Wadsworth, ${ }^{1} \mathrm{C}$ Kaltsidis, ${ }^{3}$ E S Shearer, 'L Dwyer, ${ }^{2} \mathrm{G} J \mathrm{M}$ Webster, ${ }^{1} \mathrm{R}$ Sturgess. 'Digestive Diseases Unit, Aintree University Hospital, Liverpool; '2Department of Gastroenterology, University College London Hospitals, London; ${ }^{3}$ Anaesthesia and Critical Care, Aintree University Hospital NHS Foundation Trust, Liverpool, UK

Introduction Although conventional endoscopic techniques for the removal of stones from the biliary tree are highly effective, they fail in up to $10 \%$ of patients with choledocholithiasis. With the introduction of single operator peroral cholangioscope (POC), the SpyGlass ${ }^{\circledR}$ System, stone fragmentation under direct visual control has proven to be highly effective and is now emerging as an important endoscopic therapy. We describe the characteristics and outcomes of patients undergoing POC directed electrohydraulic lithotripsy (POC-EHL) in two tertiary Hepatobiliary units in England.

Methods Details of all patients undergoing POC-EHL at Aintree University Hospital and University College London Hospitals were prospectively recorded. Data collected included demographics, number of ERCPs, site of the stone, number of POC-EHL sessions, success of stone clearance and complications.

Results A total of 93 patients were referred for POC-EHL. There were 25 males $(27 \%)$ and 68 females (73\%). The median age was 65 (20-92) years. $71(76 \%)$ patients were tertiary referrals. $62(67 \%)$ patients had at least two or more endoscopic attempts at stone removal prior to referral for POC-EHL. In six patients POC-EHL was not required because at ERCP prior to POC, the ducts could be cleared with conventional techniques. In five patients EHL was not attempted due to the size, configuration and quantity of stones. With the knowledge that these patients were fit for cholecystectomy, they were referred for cholecystectomy and bile duct exploration as a one-stage procedure. All POC-EHL sessions were performed under general anaesthesia. Of the 82 patients undergoing POC-EHL $61(75 \%)$ patients needed one POC-EHL session and $10(12 \%)$ required two sessions and $6(7 \%)$ required three sessions for complete stone extraction. In 5/82 (6\%) complete stone extraction was not possible despite POC-EHL and these patients were referred for surgery. The sites of stones were common bile duct in $48 \%$, cystic duct and $\mathrm{CBD}$ in $20 \%$, cystic duct in $4 \%$, common hepatic duct in $10 \%$ and intra-hepatic ducts in $18 \%$. Three patients developed cholangitis post POC-EHL, responding to antimicrobial therapy. Two patients experienced post-procedure bleeding, only one patient required endoscopic intervention.

Conclusion On an intention to treat basis, $89 \%$ of patients referred for POC-EHL were treated successfully. Successful POC-EHL frequently requires combination with other stone removal techniques including mechanical lithotripsy. POC-EHL appears to be a safe and effective technique in the clearance of refractory biliary stones.

Disclosure of Interest None Declared.

\section{PTH-067 YIELD OF ENDOSCOPIC ULTRASOUND (EUS) IN PATIENTS WITH DILATED COMMON BILE DUCT (CBD) AND OR PANCREATIC DUCT (PD) WITH NORMAL LIVER FUNCTION TEST (LFTS) AND CROSS-SECTIONAL IMAGING}

doi:10.1136/gutjnl-2013-304907.554

1."V Mitra, 'M Nayar, ' $S$ Bonnington, ${ }^{2} \mathrm{~J}$ Scott, ${ }^{2} \mathrm{~K}$ Anderson, ${ }^{1} \mathrm{R}$ Charnley, ${ }^{1} \mathrm{~B}$ Jaques, ${ }^{1} \mathrm{G}$ Sen, 'S White, 'D Manas, '1J French, ' $\mathrm{K}$ Oppong. 'HPB; ' ${ }^{2}$ Radiology, FREEMAN HOSPITAL, Newcastle, UK

Introduction The finding of dilated $\mathrm{CBD}$ or $\mathrm{PD}$ or both (double duct sign) on abdominal cross-sectional imaging (CT or MRI) in patients with normal LFTs frequently leads to further investigation by EUS if a clear cause is not demonstrated on imaging. There is limited literature on the yield of EUS in this setting.

Methods A retrospective review of our prospectively maintained EUS database was carried out to identify patients who underwent EUS for dilated duct(s), normal LFTs and non-diagnostic cross-sectional imaging between January 2007 and August 2011. Our aim was to evaluate the yield of EUS in this setting. Minimum follow up was for 12 months.

Results 83 patients ( $3 \%$ of pancreaticobiliary EUS procedures during this period) were identified. Mean age was 66.7 years $(30-87)$. There was a female preponderance (73\%). 40(48\%) had dilated CBD only, $5(6 \%)$ had solitary PD dilatation and $38(46 \%)$ had both CBD and $\mathrm{PD}$ dilatation on prior imaging. EUS was concordant with prior imaging in $60(72 \%)$ patients and discordant in $23(28 \%)$ [partial agreement in 18, non-dilated ducts in 3 and different duct dilated in 2]. $16(19 \%)$ patients had a new finding on EUS [ 3 cbd stones, $3 \mathrm{cbd}$ polyps, 4 microlithiasis, 1 ampullary adenoma, 3 chronic pancreatitis, 1 pancreatic duct adenocarcinoma (PDAC) and 1 portal vein compressing mid CBD]. Of these $11(13 \%)$ were felt to be the cause of duct dilatation. On subsequent MDT review, the PDAC was identified on the initial scan from the referring hospital. 45\% (10/22) of males compared to $9.8 \%$ of $(6 / 61)$ females had a new finding on EUS ( $p=0.0008)$. Overall, more females had symptoms compared to males $(p=0.065)$. There was no significant association between symptoms and new finding on EUS $(p=0.24) .7(17.5 \%)$ of the isolated $\mathrm{CBD}$ dilatation, $3(60 \%)$ of the isolated $\mathrm{PD}$ dilatation and $1(2.5 \%)$ of the dilated $\mathrm{CBD}$ and $\mathrm{PD}$ had a causative diagnosis respectively. Follow up of patients with no cause of duct dilatation on EUS revealed that 18 patients $(54.5 \%)$ in the dilated CBD cohort had prior cholecystectomy [one patient had peri-ampullary diverticulum and prior cholecystectomy] while 2 and 10 patients in the dilated CBD and PD cohort $(n=37)$ had ampullary stenosis and prior cholecystectomy respectively.

Conclusion Our study shows that EUS has a significant yield in individuals with unexplained duct dilatation and normal LFT $(13 \%$ had a causative diagnosis). The yield was highest in isolated PD dilatation. A new finding was significantly more likely in men then women. EUS should ideally follow review of original cross sectional imaging by a HPB radiologist.

Disclosure of Interest None Declared.

\section{PTH-068 THE USE OF OESOPHAGEAL CAPSULE ENDOSCOPY IN PATIENTS WITH HAEMOPHILIA; EXPERIENCE FROM A TERTIARY CENTRE}

doi:10.1136/gutjnl-2013-304907.555

1, ${ }^{\prime} \mathrm{Y}$ L Ang, ${ }^{2} \mathrm{~A}$ Koulaouzidis, ${ }^{2} \mathrm{~S}$ Douglas, ${ }^{1,2 \mathrm{~J}} \mathrm{~N}$ Plevris. ${ }^{1}$ Medical School, The University of Edinburgh; ${ }^{2}$ Centre for Liver \& Digestive Disorders, The Royal Infirmary of Edinburgh, Edinburgh, UK

Introduction A great proportion of haemophiliacs are considered at risk of being co-infected with hepatitis $\mathrm{C}(\mathrm{HCV})$ and variant Creutzfeldt-Jakob Disease (vCJD). ${ }^{1}$ Chronic hepatitis $\mathrm{C}$ leads to liver cirrhosis, which in turn causes portal hypertension and varices. 2 Alternative endoscopic modalities have been developed for the investigation of the upper gastrointestinal (GI) tract, such as oesophageal capsule endoscopy (OCE). However, OCE is widely accepted and its indications are still under evaluation. ${ }^{3}$ Our aim was to evaluate the use of OCE in a tertiary referral centre for GI problems in Lothian, Southeast Scotland, giving a special focus on OCE in haemophiliacs.

Methods A retrospective review of the OCE database from May 2005 to March 2012. Electronic case notes and OCE reports were reviewed. Demographics and clinical background, in particular haemophilia, hepatitis C, HIV and cirrhosis, reason for referral and OCE findings were abstracted. 